\title{
Derecho al acceso a tratamientos y medicamentos experimentales, caso Estados Unidos-Colombia: críticas a su traslación al ordenamiento interno
}

\author{
Right to Access to Experimental \\ Treatments and Drugs, US-Colombia \\ Case: Criticisms of its Translation into \\ the Domestic System
}

Direito ao acesso a tratamentos experimentais e medicamentos, caso Estados Unidos-Colômbia: crítica à sua tradução para o direito interno 


\section{Resumen}

En el presente artículo de investigación se analiza la incorporación del derecho a acceder a tratamientos experimentales en Estados Unidos y Colombia, examinando su inserción jurisprudencial, sus elementos esenciales y las regulaciones que cada país establece frente a la provisión de estos procedimientos y medicinas no convencionales. Posteriormente, se plantea una serie de críticas a la migración o la traslación que la Corte Constitucional realizó de este derecho desde la jurisprudencia y la legislación norteamericana al sistema de salud colombiano. El fin de este texto académico es realizar un análisis comparativo de ambos ordenamientos sanitarios, bajo una mirada crítica que contraste el rol del Estado y los actores de la salud (fabricantes, aseguradoras, pacientes) frente al acceso de las medicinas y los procedimientos en fase de investigación o experimentación.

\section{Palabras clave}

Derecho a tratamientos experimentales; actores de la salud; análisis comparativo; críticas a la traslación del derecho; regulación de medicamentos o procedimientos en investigación; rol del Estado y los particulares. 


\section{Abstract}

This research article analyzes the incorporation of the right to access to experimental treatments in the United States of America and Colombia. It also examines its case-law insertion, essential elements, and the regulations that each country has established for the provision of these unconventional procedures and drugs. Subsequently, it makes a series of criticisms over how the Constitutional Court has migrated or translated this right from US case law and legislation to the Colombian health care system. Therefore, this paper intends to carry out a comparative analysis of both health care systems from the perspective of the role of both the State and health actors (manufacturers, insurers, patients) in accessing experimental drugs and procedures.

\section{Keywords}

Right to experimental treatments; health actors; comparative analysis; criticism of the translation of law; regulation of experimental drugs or procedures; role of the State and individuals. 


\section{Resumo}

Este artigo de pesquisa analisa a incorporação do direito de acesso a tratamentos experimentais nos Estados Unidos e na Colômbia, examinando sua inserção jurisprudencial, seus elementos essenciais e as regulamentações que cada país estabelece diante da provisão desses procedimentos e medicamentos não convencionais. Em seguida, uma série de críticas à migração ou ao deslocamento que a Corte Constitucional realizou desse direito e da lei dos Estados Unidos para o sistema de saúde colombiano. O objetivo deste texto acadêmico é realizar uma análise comparativa de ambos os sistemas de saúde, numa perspectiva crítica que contrasta o papel do Estado e dos atores de saúde (fabricantes, seguradoras, pacientes) ante o acesso a medicamentos e aos procedimentos em fase de pesquisa ou experimentação.

\section{Palavras-chave}

Direito a tratamentos experimentais; atores de saúde, análise comparativa; críticas à tradução da lei; regulação de medicamentos ou procedimentos em investigação; papel do Estado e dos indivíduos. 
Sumario: Introducción. 1. Incorporación del derecho a tratamientos experimentales en Estados Unidos. 1.1 Introducción del derecho en la jurisprudencia. 1.2 Elementos esenciales. 1.3 Regulación en los Estados Federales. 2. Incorporación del derecho a tratamientos experimentales en Colombia. 2.1 Introducción del derecho en la jurisprudencia. 2.2 Elementos esenciales. 2.3 Regulación interna. 3. Críticas a la inserción del derecho a tratamientos experimentales, por parte de la Corte Constitucional, al sistema de salud colombiano. 3.1. Valoración del acceso a tratamientos experimentales bajo la perspectiva de un derecho positivo, cuando en Estados Unidos se introdujo como un derecho negativo. 3.2 Concepción del derecho bajo un criterio solidario, cuando en la perspectiva norteamericana se planteó con un enfoque individualista. 3.3 Inclusión del derecho en el marco de un enfoque de intermediación que se opone al modelo de independencia médica norteamericano. 3.4 Introducción del derecho en el contexto de un mercado regulado, cuando en Estados Unidos se incluyó en un mercado desregulado. Conclusiones. Bibliografía.

\section{Introducción}

El derecho a tratamientos experimentales fue introducido en Colombia mediante la Sentencia T-057 de 2015, ${ }^{1}$ extrapolando una decisión proferida en el 2007 por la Corte de Apelaciones de Columbia, Estados Unidos, en la cual se señaló que no era posible que el Estado se opusiera al acceso a estos procedimientos, pues ello constituía una violación de la esfera privada de libertad de los individuos, que no se compaginaba con los principios tutelares de la Constitución Política Norteamericana. ${ }^{2}$ Sin embargo, bajo nuestra perspectiva, la inserción jurisprudencial del derecho dentro del ordenamiento colombiano se realizó de forma inadecuada, pues el sistema jurídico nacional no tuvo en cuenta las bases iusfilosóficas que permitieron su inclusión en el sistema norteamericano.

El presente artículo de investigación tiene como eje central analizar a profusión un conjunto de críticas a la traslación del derecho a tratamientos experimentales desde el sistema jurídico estadounidense al modelo sanitario colombiano.

El abordaje de estas críticas requiere necesariamente comprender la incorporación del derecho en cada uno de los sistemas jurídicos, para lo cual se formulará un primer apartado en el que se examinará su inserción dentro de la jurisprudencia norteamericana, sus aspectos fundamentales y su inclusión en las leyes federales estadounidenses. En un segundo apartado se abordará la incorporación del derecho en la jurisprudencia colombiana, sus elementos esenciales y su regulación a nivel nacional. En un tercer apartado se plantean las críticas a la inserción del derecho al sistema de salud nacional. Finalmente, se elabora un conjunto de conclusiones que permiten sintetizar los hallazgos de la investigación.

1 Corte Constitucional, Sentencia T-057 de 2015. M. P. Martha Victoria Sáchica Méndez.

2 Corte de Apelaciones del Distrito de Columbia, caso Abigail Alliance for Better Access to Developmental Drugs and Washington Legal Foundation, appellants vs. Eschenbach, sentencia del 7 agosto de 2007. 


\section{Incorporación del derecho a tratamientos experimentales en Estados Unidos}

El sistema de salud norteamericano tiene un enfoque privatista, ${ }^{3}$ con fundamento en la Enmienda XIV de la Constitución Política de ese país, ${ }^{4}$ que ampara la libertad de los usuarios de adoptar un seguro médico para acceder a medicamentos convencionales o experimentales y que limita la competencia del Ejecutivo para establecer regulaciones a los fabricantes o las empresas prestadoras del servicio médico. La asistencia privada se articula a través de pólizas de salud individuales, directas o gestionadas por agrupaciones de proveedores, que son sufragadas por los empleadores o por el propio interesado, mientras que las coberturas médicas que garantiza el Gobierno Federal se canalizan a través del programa Medicare, ${ }^{5}$ que cubre únicamente a los estadounidenses de más de 65 años de edad, a personas con discapacidades, a los veteranos excombatientes y a los nativos. ${ }^{6}$

En Estados Unidos existe una política de incentivos fiscales para promover el aseguramiento privado de las personas, de manera que los empleadores que vinculen a sus trabajadores a coberturas médicas, así sea para medicamentos experimentales, puedan recibir una exención de su carga tributaria. ${ }^{7}$ Asimismo, se conceden créditos fiscales (tax credits) para aquellas personas cuyos ingresos se encuentren entre el 100 y el $400 \%$ del umbral federal de pobreza, ${ }^{8}$ lo que permite al Estado girar directamente estos recursos a la compañía aseguradora del contribuyente, y estos se deducen de la prima del seguro que el ciudadano deba pagar. ${ }^{9}$

3 El $70 \%$ de la población de Estados Unidos está cubierta por un seguro de salud privado. La cobertura se garantiza a través de un contrato civil, que se rige por la autonomía de la voluntad y tiene muy poco control gubernamental. La única regulación estatal que existe es sobre las prestaciones que deben estar obligatoriamente cubiertas (Celia Iriart, "El sistema de salud de los Estados Unidos: mitos y realidades", Revista Saúde em Redes 1 (2016), p. 13).

4 Enmienda XIV de la Constitución Política de Estados Unidos. “Todas las personas nacidas o naturalizadas en los Estados Unidos y sometidas a su jurisdicción son ciudadanos de los Estados Unidos y de los Estados en que residen. Ningún Estado podrá dictar ni dar efecto a cualquier ley que limite los privilegios o inmunidades de los ciudadanos de los Estados Unidos; tampoco podrá Estado alguno privar a cualquier persona de la vida, la libertad o la propiedad sin el debido proceso legal; ni negar a cualquier persona que se encuentre dentro de sus límites jurisdiccionales la protección de las leyes, igual para todos".

$5 \quad$ Este plan se divide en dos partes: la parte A, que paga los beneficios a los hospitales y el cuidado domiciliario calificado de enfermería, y la parte B, que contribuye a pagar las cuentas médicas y los servicios ambulatorios. Esta cobertura es opcional y requiere el pago de una prima mensual (Augustus Trajano y Sonia Fleury, "La reforma del sistema de salud de los Estados Unidos de América en los años 90", Revista Salud Colectiva 1 (2005), p. 138).

6 Organización Panamericana de la Salud, Perfil de sistemas y servicios de salud en Estados Unidos de América, Columbia, Editorial Panamericana, 2018, p. 110.

7 Alejandro Arrieta, "Seguro de salud y principio contributivo de la seguridad social en los Estados Unidos de América", Revista Latinoamericana de Derecho Social 23 (2016), p. 7.

$8 \quad$ El umbral de pobreza es una medida del nivel de ingresos emitida anualmente por el Departamento de Salud y Servicios Humanos, que se utiliza para determinar la elegibilidad de las personas para ciertos programas y beneficios, incluyendo ahorros en el mercado de seguros médicos.

9 Ana Belén Macho, "La reforma de la financiación del sistema sanitario en Estados Unidos. ¿Es la affordable care act ("obamacare") relevante para Europa?", Tax and Public Finance Law Seminar Series 1 (2015), p. 10. 
El esquema sanitario estadounidense incorpora un derecho de libertad económica para los particulares, que impide que el Estado pueda interferir en la comercialización o distribución de fármacos que efectúan los agentes empresariales, y en la decisión de los pacientes de usarlos para sus necesidades, salvo que una ley de la república ordene la intervención federal de ese mercado. ${ }^{10}$

\subsection{Introducción del derecho en la jurisprudencia}

El tráfico de bienes y servicios sanitarios dentro del mercado norteamericano se encuentra someramente regulado por la Food and Drug Administration (FDA), a través de una lista pública ${ }^{11}$ en la cual incorpora los fármacos aprobados sobre la base de inocuidad y eficacia, y descarta los medicamentos que se han descontinuado, los que comporten riesgos demostrados para los usuarios o los que no evidencien un grado de utilidad. ${ }^{12}$

Tradicionalmente, la FDA había rechazado la aprobación de medicamentos o procedimientos experimentales que no hubiesen transitado las diferentes fases de investigación para ser aprobados, ${ }^{13}$ por no tener un grado de eficacia acreditable sobre sus riesgos o efectos para los pacientes que los requerían.

Sin embargo, la Corte de Apelaciones del Distrito de Columbia, mediante decisión del 7 de agosto de 2007, estableció oficialmente el derecho al acceso a tratamientos experimentales a partir del cual se determinó que pacientes afectados por una enfermedad terminal no fueran obligados a esperar hasta que se surtiera el trámite de aprobación por parte de la FDA para poder acceder a un medicamento que les prescribiera su médico tratante, de manera que pudieran elegir de forma autónoma si se sujetaban a la práctica de estos procedimientos, después de habérseles informado los riesgos y beneficios que

10 Los derechos de libertad se relacionan con la prohibición de interferencia indebida en la esfera particular de su titular, ya sea derivada de acciones del poder público o de sujetos privados. De esa forma, se impone una conducta omisiva por parte del Estado y los particulares (Keith Banting y Stand Corbett, Health Policy and Federalism. A comparative perspective on Multi-Level Governance, New York, Queen's University, 2002, p. 36).

11 La lista se denomina "Productos farmacéuticos aprobados con evaluaciones de equivalencias terapéuticas", conocida como el Libro Anaranjado, en el cual se enumeran más de 10.300 medicamentos aprobados.

12 Robert Lowes, "Right-to-try gains steam as FDA eases compassionate use", Medscape Medical News, 2017, en https://www.medscape.com/viewarticle/886525, fecha de consulta: 17 de enero de 2020.

13 Para la autorización de fármacos por la FDA, se deben surtir varias etapas: 1) etapa preclínica: en esta etapa se hacen pruebas para analizar los componentes químicos del medicamento y se realizan exámenes en especies animales para determinar su toxicidad. Después de esta etapa, el medicamento es declarado como "nuevo fármaco en investigación". 2) etapa clínica: se trata de los procesos de evaluación del medicamento para seres humanos, a partir de tres fases: a) fase I: tiene como objetivo conocer acerca de las características propias del producto investigado y establecer los rangos de dosis aceptables, para lo cual se efectúan pruebas en voluntarios sanos y no en sujetos enfermos que podrían confundir la interpretación de las pruebas; b) fase II: se busca tener una primera aproximación de la eficacia terapéutica del fármaco y conocer más acerca del rango de dosis útiles, tolerabilidad y seguridad del producto; por tanto, en esta fase de la investigación participan pacientes voluntarios que padecen la enfermedad sobre la cual se espera que actúe el fármaco estudiado; c) fase III: se compara la eficacia del medicamento experimental con otro fármaco ya aprobado, a través de pruebas científicas que se practican a una multiplicidad de voluntarios afectados por la patología que se pretende contrarrestar (Carlos Bustamante Rojas, Fases del desarrollo de un nuevo medicamento. Ponencia del Programa de Especialización en Farmacología Clínica, Bogotá, Universidad de La Sabana, 2013, p. 5). 
podría suponer su aplicación. De esa forma, se confirió un derecho negativo al usuario y, correlativamente, se impuso un deber de abstención al Estado para no perturbar o impedir la circulación de estos medicamentos.

Esta decisión constituyó un hito trascendental, en la medida que limitó el poder del Estado norteamericano para interferir en la libertad de los actores que integran el mercado de la salud, lo que permitió que los fabricantes pudieran ofrecer medicamentos o servicios experimentales que hubiesen completado la fase I de investigación clínica, ${ }^{14}$ y que los usuarios pudieran adquirirlos, según su capacidad económica y conforme a las órdenes de su médico tratante, sin las barreras impuestas por la FDA.

\subsection{Elementos esenciales}

El derecho a acceder a medicinas experimentales tiene como eje tutelar proteger la esfera íntima de las personas, como ámbito irreductible que no puede ser afectado por decisiones administrativas, de manera que los usuarios sean los que decidan si corren o no los riesgos que su suministro puede comportar, pues otra interpretación sería incompatible con la tradición libertaria y privatista de la nación estadounidense, que incluso permite que los individuos empleen los medios que consideren convenientes para salvar sus vidas, así impliquen aleas desmesurados o desproporcionados. ${ }^{15}$

La reflexión de la Corte de Apelaciones de Columbia parte, entonces, de la premisa según la cual, ante el "riesgo cierto y concreto" que representan las patologías en las personas, el Estado no puede obstaculizar la atención médica sobre la base de efectos "indeterminados o imprecisos" del medicamento experimental.

El acceso a este derecho imposibilita al Estado para prohibir estos procedimientos, en la medida que representan la única posibilidad de supervivencia de un paciente, al no existir alternativa terapéutica dentro del sistema de salud que sea apropiada para sus dolencias, pues el paciente tiene derecho a "intentar" su recuperación, aunque eventualmente el tratamiento no tenga éxito ni sea eficaz, porque esa tentativa es preferible a permanecer pasivo, ante el paso inexorable de la enfermedad. ${ }^{16}$

14 Un estudio en fase I generalmente implica que se han superado las pruebas del medicamento con animales. Para realizar este tipo de ensayos, se deben convocar de 20 a 80 sujetos, para determinar el metabolismo y las acciones del fármaco en humanos, los efectos secundarios asociados con el aumento de dosis y, de ser posible, obtener evidencia temprana sobre la efectividad (Julie Turkewitz, "Patients Seek 'Right to Try' New Drugs", The New York Times, 2015, en https://www.nytimes.com/2015/01/11/us/patients-seek-right-to-try-new-drugs. html, fecha de consulta: 17 de enero de 2020).

15 La Patient Self Determination Act de 1990 reconoce la libertad de los seres humanos sobre su propia persona, a partir de la cual pueden decidir sobre el tipo de tratamientos o intervenciones que pueden recibir en los hospitales y centros sanitarios.

16 Emily Hogan, "Legislación del derecho intentar y sus implicaciones para el proceso de aprobación de drogas de la FDA", Revista de Derecho y Política 50 (2016), p. 175. 
La incorporación del derecho a acceder a tratamientos experimentales no se quedó solo en los tribunales de justicia, pues se comenzó a ver la necesidad de disponer de un instrumento legal mediante el cual las personas pudieran exigir ese derecho sin tener que acudir a los jueces, por lo cual varios estados federales comenzaron a aprobar en sus legislaciones la posibilidad de acceder a estos insumos médicos.

\subsection{Regulación en los estados federales}

La ley que abrió el camino para que los enfermos pudieran obtener este tipo de medicamentos fue promulgada por el gobernador de Colorado, el 17 de mayo de 2014. ${ }^{17}$ Mediante esta legislación se pretendió reducir el tiempo que los pacientes debían esperar para que les fuera suministrado un medicamento, pues se tenían estimaciones de que el proceso completo de aprobación por parte de la FDA podía extenderse entre 10 y 15 años. ${ }^{18}$ También se quiso impedir que las personas con enfermedades terminales tuvieran que soportar largos años para acceder a medicamentos en fase de investigación que habían sido prescritos por su médico tratante.

En el marco de esta regulación, se indicó que para que una persona sea elegible para acceder a tratamientos experimentales, debía cumplir las siguientes condiciones:

- Presentar una enfermedad terminal determinada por el profesional de la salud que lleva su proceso epidemiológico.

- Gue el galeno que atiende el caso, después de estudiar todas las opciones de tratamientos aprobados por la FDA, considere que el medicamento o tratamiento en investigación es el adecuado para tratar su patología.

- Gue preste su consentimiento por escrito, salvo que carezca de la capacidad mental para manifestarlo, caso en el cual los representantes legales procederán a emitir una autorización sustitutiva.

El rol del fabricante se encuentra ampliamente protegido mediante esta legislación, pues se le garantiza la posibilidad de ofrecer el medicamento de forma gratuita o exigiendo los costos derivados de su producción y distribución. La única manera en que se obliga a la farmacéutica a pagar los gastos es cuando celebra un contrato con el paciente para sufragarlos.

Frente a las empresas aseguradoras de salud, la reforma legal establece la exención del pago por el uso de estos medicamentos experimentales, salvo que

17 Colorado House Bill 14-1281. Reforma legal “sobre la autorización para que los enfermos terminales tengan acceso a los productos de investigación que no han sido aprobados por la Administración de Alimentos y Medicamentos Federal y para que los pacientes puedan participar en ensayos clínicos", publicada el 17 de mayo de 2014

18 Jann Bellamy, “The illusions of 'right to try' laws", Review Science-Based Medicine, 2014, en https://sciencebasedmedicine.org/the-illusions-of-right-to-try-laws/, fecha de consulta: 17 de enero de 2020. 
ese deber se hubiese incorporado mediante una póliza contractual celebrada entre la entidad y el paciente. En ese sentido, la orden para que se practique el tratamiento no puede entenderse como una nueva cobertura que deba garantizarse con los recursos derivados de las primas de aseguramiento.

La finalidad de la ley de Colorado es que los insumos experimentales no se financien con las sumas que las personas cotizan para financiar la seguridad social, sino que se subvencionen con los recursos propios del paciente o su familia bajo la óptica de una filosofía individualista, lo cual evita que el Estado y las entidades aseguradoras tengan que sufragarlos imperativamente con cargo a su presupuesto. ${ }^{19}$

Después de la ley de Colorado de 2014, se sumaron a la aprobación de tratamientos experimentales para personas en situación crítica 38 estados federales, y quedaron pendientes solo 13 de los demás gobiernos locales. ${ }^{20} \mathrm{Al}$ extenderse la aplicación de esta reforma a múltiples ciudades del país, y con el cambio de Gobierno encabezado por Donald Trump, caracterizado por la defensa de la libertad comercial y la autotutela de los pacientes, empezó a visibilizarse que era necesario introducir una legislación uniforme que permitiera garantizar el acceso al derecho a todos los ciudadanos, lo que implicó finalmente la aprobación, en agosto de 2017, del Proyecto de Ley S. 204 (115), por medio del cual se establecieron las condiciones genéricas y los presupuestos para acceder a medicamentos no aprobados por la FDA.

Esta legislación replicó las condiciones establecidas en la regulación de Colorado para la concesión de los tratamientos experimentales, e hizo hincapié en que estos elementos solo podían ser suministrados después de superar un control mínimo por parte de la autoridad sanitaria, si no había posibilidad de reemplazarlos por los medicamentos convencionales aprobados por el sistema de salud, y si el médico tratante los había ordenado después de explicarle a su destinatario los efectos potencialmente nocivos o benéficos que podían generarse por su aplicación.

\section{Incorporación del derecho a tratamientos experimentales en Colombia}

Es importante comenzar señalando que, por regla general, los medicamentos $\mathrm{y}$ tratamientos que se les deben asegurar a todos los ciudadanos se encuen-

19 Michael Ciaglo, "Quicker access to experimental drugs", The New York Times, 2015, en https://www.nytimes. com/2015/02/12/opinion/quicker-access-to-experimental-drugs.html, fecha de consulta: 17 de enero de 2020.

20 Los estados federales que aprobaron esta legislación son: Alabama, Arizona, Arkansas, California, Colorado, Connecticut, Florida, Georgia, Idaho, Illinois, Indiana, Iowa, Kentucky, Luisiana, Maine, Maryland, Michigan, Minnesota, Misisipi, Missouri, Montana, Nevada, New Hampshire, Carolina del Norte, Dakota del Norte, Ohio, Oklahoma, Oregón, Pensilvania, Carolina del Sur, Dakota del Sur, Tennessee, Texas, Utah, Virginia, Virginia Occidental, Washington y Wyoming. 
tran establecidos en un plan de beneficios unificado por la Corte Constitucional mediante Sentencia T-760 de 2008. ${ }^{21}$ En este plan no se encuentran los procedimientos experimentales, de manera que las empresas prestadoras de salud (EPS) no se encuentran obligadas a financiarlos, por no estar dentro de su plataforma de servicios.

La Ley 1751 de 2015 (Ley Estatutaria de Salud) establece en su artículo 15 que no se pueden subvencionar con recursos públicos los tratamientos o medicamentos que se encuentren en fase de experimentación. ${ }^{22}$ Esta disposición es concordante con el artículo 7 del Acuerdo 008 de 1994 del Ministerio de Salud, que excluyó como prestaciones del sistema los tratamientos con drogas o sustancias experimentales para cualquier tipo de enfermedad. ${ }^{23}$

\subsection{Introducción del derecho en la jurisprudencia}

Ante la existencia de una prohibición legal que impedía que se financiaran con recursos públicos los tratamientos experimentales, se hizo necesario que la Corte Constitucional, mediante Sentencia T-057 de 2015, aprobara la prestación de estos procedimientos para garantizar la integralidad de los medicamentos que una paciente que padecía un estado vegetativo requería para intentar su recuperación o la paliación de sus dolores, pues su EPS se negaba a realizar el procedimiento, alegando que su naturaleza experimental lo excluía del plan de beneficios. ${ }^{24}$

La alta corporación, en su decisión, autorizó el procedimiento, en el entendido de que las exclusiones del sistema no podían desconocer el derecho de los pacientes a gozar de la integralidad de las prestaciones que buscaban la satisfacción plena de su derecho a la salud. ${ }^{25}$ Asimismo, señaló que no podía restringirse la autonomía de los usuarios para optar por los elementos médicos que consideran necesarios, teniendo en cuenta que constituyen su última alternativa, y que no había un procedimiento sustituto dentro del plan de beneficios para tratar la enfermedad. ${ }^{26}$

\subsection{Elementos esenciales}

La aplicación de este derecho está condicionada a que el paciente padezca una enfermedad catastrófica o terminal, pues esta situación obliga al sistema

21 Corte Constitucional, Sentencia T-760 de 2008, M. P. Manuel José Cepeda Espinosa.

22 Congreso de la República, Ley 1751 de 2015, "Por medio de la cual se regula el derecho fundamental a la salud y se dictan otras disposiciones", Diario Oficial 49.427, de 16 de febrero de 2015.

23 Ministerio de Salud, Acuerdo 008 de 1994, "Por el cual se adopta el Plan Obligatorio de Salud para el Régimen Contributivo del Sistema General de Seguridad Social en Salud".

24 Corte Constitucional, Sentencia T-057 de 2015, M. P. Martha Victoria Sáchica Méndez.

25 Según Gañan Echavarría, el derecho a la salud tiene como propósito garantizar a todas las personas el diagnóstico, el tratamiento y la rehabilitación, para recuperarse de sus dolencias físicas o psicológicas y mejorar su calidad de vida, mediante servicios médicos integrales (Jaime León Gañan Echavarría, “De la naturaleza jurídica del derecho a la salud en Colombia", Revista RIDE de la Biblioteca digital de la Superintendencia de Salud 3 [2013], p. 8). 
de salud a garantizar un tratamiento integral, oportuno, continuo e informado, y compele a las entidades a suministrar todos los medios posibles, así sea experimentales, para paliar el padecimiento intrínseco a estas patologías. ${ }^{27}$

La concesión de estos procedimientos no convencionales está supeditada a que no se pueda reemplazar el tratamiento por cualquiera de los establecidos en el plan de beneficios, por lo que el paciente debe demostrar su falta de capacidad de pago total o parcial, ya sea mediante un balance certificado por contador, a través de la declaración de renta, o también acreditando la falta de mecanismos alternativos de protección, como pólizas voluntarias de salud o contratos de medicina prepagada. ${ }^{28}$

Después de que se compruebe la falta de solvencia económica, el juez puede inaplicar las restricciones de la Ley 1751 de 2015, y procede a autorizar el tratamiento experimental, siempre y cuando no exista una vía alternativa eficaz para su obtención en el momento oportuno. ${ }^{29} \mathrm{El}$ financiamiento de los tratamientos experimentales con cargo a los recursos públicos tiene como fundamento el principio de solidaridad a partir del cual el Estado debe subvencionar los medicamentos o procedimientos que requieren las personas, si no pueden hacerlo por su propia cuenta. ${ }^{30}$ Esta contribución solidaria tiene como base la colaboración que hace la sociedad para aportar todos los medios necesarios para el suministro de las prestaciones experimentales a quienes eventualmente puedan necesitarlas y no tengan cómo costearlas. ${ }^{31}$

Por otra parte, la jurisprudencia de la Corte Constitucional ha señalado que el acceso a los tratamientos de esta naturaleza requiere de la aceptación expresa o el consentimiento por parte del paciente y de los riesgos concretos y precisos a los que puede estar sujeto. ${ }^{32} \mathrm{Si}$ la persona no se encuentra en un estado mental idóneo para expresar su asentimiento, por padecer una discapacidad mental o un estado de inconciencia que le impida discernir, se puede aceptar la manifestación de un consentimiento sustitutivo en cabeza de las personas responsables de quien no es capaz de decidir por sí mismo. ${ }^{33}$

27 Mateo Duque Giraldo, "Precedente constitucional en materia de derecho a ser 'intentado' o right to try para tratamientos experimentales", Revista CES de Derecho 1 (2018), p. 153.

28 Si la persona acredita estos elementos, existe la obligación de garantizar su atención o la de sus beneficiarios por las instituciones públicas prestadoras de servicios de salud o por aquellas privadas con las cuales el Estado tenga contrato.

29 Corte Constitucional, Auto 035 de 2009, M. P. Manuel José Cepeda Espinosa.

30 Carlos Miguel Herrera, "El concepto de solidaridad y sus problemas político-constitucionales. Una perspectiva iusfilosófica", Revista de Estudios Sociales 46 (2013), p. 64.

31 Leonardo Cañón Ortegón, “La solidaridad como fundamento del Estado social de derecho, de la seguridad social y la protección social en Colombia", Revista Páginas de Seguridad Social 1 (2017), p. 9.

32 Corte Constitucional, Sentencia T-180 de 2013, M. P. Jorge Ignacio Pretelt Chaljub.

33 Duque Giraldo, "Precedente constitucional en materia de 'derecho a ser intentado' o 'right to try' para tratamientos experimentales", op. cit., p. 154. 
Al tratarse de una intervención experimental que implica mayor compromiso con la integridad física del afectado y que le genera una gran incertidumbre sobre el beneficio esperado, el personal de salud debe garantizar un consentimiento de carácter cualificado. ${ }^{34} \mathrm{El}$ alcance del consentimiento debe ser especialmente riguroso, para evitar que los pacientes sean instrumentalizados y puedan prestar su anuencia sin ninguna coacción o engaño. ${ }^{35}$

La autonomía del usuario tiene un alcance tan profundo, que incluso le permite asentir la aplicación de procedimientos que no impliquen expectativas reales de mejoría o que incluso lo pueden poner el peligro, siempre que no comprometan derechos de terceros. Desde esa perspectiva, existe la obligación para los profesionales de la salud de brindar el "chance u oportunidad" 36 de mejoría a sus pacientes, mediante la aplicación de tratamientos experimentales; queda en manos del usuario valorar de manera informada los aspectos positivos y negativos del procedimiento.

Por otra parte, es importante señalar que la concesión de tratamientos experimentales se encuentra sujeta a una intermediación que limita la facultad de ordenación del médico tratante, pues existe una burocracia técnica que limita la autonomía de los médicos para prescribir este tipo de fórmulas clínicas. La Resolución 1328 de 2016 establece que unas juntas médicas adscritas a las EPS tienen la potestad de aprobar o desaprobar el acceso a estos procedimientos, que se encuentran excluidos del plan de beneficios. ${ }^{37}$

Por lo regular, las juntas médicas niegan el acceso a los medicamentos experimentales, en desconocimiento de la autonomía médica de los profesionales de la salud, lo que afecta el acceso de los pacientes a esos servicios médicos. ${ }^{38}$

\subsection{Regulación interna}

El derecho a acceder a tratamientos experimentales no se encuentra legislado dentro del ordenamiento colombiano, pues no existe una norma con fuerza de

34 Corte Constitucional, Sentencia T-303 de 2016, M. P. Jorge Ignacio Pretelt Chaljub.

35 Según el artículo 7 del Pacto de Derechos Civiles y Políticos de las Naciones Unidas, nadie puede ser sometido sin su libre consentimiento a experimentos médicos o científicos (Naciones Unidas, Pacto de Derechos Civiles y Políticos. Ratificado por la Asamblea General en su Resolución 2200 A (XXI) de 16 de diciembre de 1966).

36 Corte Constitucional, Sentencia T-901 de 2009, M. P. Humberto Antonio Sierra Porto.

37 Artículo 12 de la Resolución 1328 de 2016. “Cuando el profesional de la salud tratante prescriba alguno de los servicios o tecnologías explícitamente excluidas, deberá consultar en cada caso particular, la pertinencia de su utilización, a la Junta de Profesionales de la Salud" (énfasis agregado) (Ministerio de Salud y Protección Social, Resolución 1328 de 2016, “Por la cual se establece el procedimiento de acceso, reporte de prescripción, garantía del suministro, verificación, control, pago y análisis de la información de servicios y tecnologías en salud no cubiertas por el Plan de Beneficios en Salud con cargo a la UPC y se dictan otras disposiciones", en https://www.minsalud.gov.co/Normatividad_Nuevo/Resoluci\%C3\%B3n\%201328\%20de\%202016.pdf, fecha de consulta: 17 de enero de 2020.

38 Andrés Ramírez, David Beltrán Rocha, Luis Suárez Durango y Sara Álvarez Rodríguez, “Implicaciones de la Ley estatutaria 1751 de 2015 sobre las entidades promotoras de salud del régimen contributivo", Revista CES de Derecho 2 (2016), p. 22. 
ley que se ocupe de reglamentar la investigación experimental o los ensayos clínicos. ${ }^{39}$ Solamente la Resolución 8430 de 1993, expedida por el Ministerio de Salud, fija unos parámetros mínimos sobre las personas que pueden ser elegibles, las condiciones en las cuales se deben practicar estos procedimientos y los principios de bioética que deben respetarse para su aplicación. ${ }^{40}$

En esta regulación se establece que en los procesos de investigación médica se le debe dar prioridad al respeto por la dignidad humana, la protección de los derechos de los sujetos y el logro de su bienestar, y se consagran unos comités de investigación que deben evaluar la eficacia y la seguridad de los procedimientos que se emplean. ${ }^{41}$

Igualmente, la norma establece un criterio amplio de legitimación que facilita la participación de cualquier persona en estos tratamientos experimentales, lo que resulta bastante riesgoso para los pacientes, ya que hay grupos específicos como las mujeres en embarazo, los menores, las personas de la tercera edad y los individuos en condición de discapacidad que deberían tener un tratamiento diferenciado y más cuidadoso frente a estos procedimientos.

La Resolución 8430 de 1993 es una regulación anacrónica y defectuosa, que demuestra la existencia de un vacío regulatorio sobre esta materia que fue omitido por la Corte Constitucional al incorporar el derecho a acceder a tratamientos experimentales dentro del sistema colombiano, en la medida que la inserción del derecho no se estructuró sobre una legislación sólida que justificara su aplicación dentro del modelo de salud nacional, como ocurrió en Estados Unidos con la ley del Estado de Colorado de 2014 y las demás legislaciones federales.

\section{Críticas a la inserción del derecho a tratamientos experimentales, por parte de la Corte Constitucional, al sistema de salud colombiano}

La introducción de derechos foráneos no es un proceso de simple subsunción silogística, sino que requiere de un análisis sistemático y teleológico de los fundamentos que facilitaron su aplicación en el contexto internacional, para ver si es posible su inserción de forma integral en la praxis local. ${ }^{42}$ En ese pun-

\footnotetext{
39 María Mónica Lopera, "Revisión comentada de la legislación colombiana en ética de la investigación en salud", Revista Biomédica 37 (2017), p. 579.

40 Ministerio de Salud, Resolución 8430 de 1993, "Por la cual se establecen las normas científicas, técnicas y administrativas para la investigación en salud", en https://www.minsalud.gov.co/sites/rid/Lists/BibliotecaDigital/RIDE/DE/DIJ/RESOLUCION-8430-DE-1993, PDF, fecha de consulta: 17 de enero de 2020.

41 Ibid., artículos 2, 3 y 4.

42 Ran Hirsch, Comparative Matters, The Renaissance of Comparative Constitutional Law, Oxford, Oxford University Press, 2014, p. 87.
} 
to, se puede retomar a Amalia Amaya, quien señala que la justificación de la aplicabilidad de un derecho por fuera de sus fronteras depende de que sea coherente con los principios del sistema constitucional en donde tiene origen, pues si no se acopla a esas pautas axiológicas, no puede regir dentro del tráfico jurídico al que se traslada. ${ }^{43}$

Según David Landau, la estrategia judicial en derecho comparado se debe apoyar en el examen de las condiciones estructurales de cada país, de manera que no se fuerce la adopción de reglas jurídicas que sean incompatibles entre los sistemas jurídicos. ${ }^{44}$

Si se analizan preliminarmente cada uno de los sistemas de salud, tanto el estadounidense como el colombiano, se pueden identificar cuatro diferencias sustantivas que la Corte Constitucional desconoció al momento de incorporar el derecho dentro del modelo sanitario nacional:

- La primera es que, en Estados Unidos, el derecho a acceder a tratamientos y medicamentos experimentales se concibe como un derecho negativo, es decir como un espacio de libertad que no puede ser interferido por el Estado que le permite a las personas acceder a bienes médicos no aprobados plenamente por la FDA; mientras tanto, en Colombia constituye un derecho fundamental autónomo, dirigido a lograr la dignidad humana y garantizar que el Estado financie tratamientos no acreditados por el sistema de salud.

- La segunda es que en Norteamérica rige una concepción individualista a partir de la cual el paciente debe financiar su tratamiento experimental, muy diferente a lo que ocurre a nivel nacional, en donde el Estado, bajo una óptica solidaria, es el que debe subvencionar económicamente la prestación de servicios médicos experimentales.

- La tercera es que a nivel nacional existe un patrón de intermediación que condiciona la exigibilidad del derecho a la aprobación de una junta médica, mientras en Estados Unidos el paciente puede ser beneficiario del tratamiento experimental únicamente con la prescripción o la orden de su médico.

- La cuarta es que en Colombia el derecho se incorporó en un mercado de la salud fuertemente regulado, caracterizado por el poder coercitivo del Estado sobre los fabricantes y comercializadores, mientras en Estados Unidos el acceso a los tratamientos experimentales tuvo como propósito que fabricantes y pacientes pudieran llegar a acuerdos privados para adquirir estos medicamentos en el contexto de un mercado desregulado.

Cada una de estas críticas, que pueden identificarse conforme a los acápites desarrollados, se analizan a continuación.

43 Amalia Amaya, "La coherencia en el derecho", en DOXA, Cuadernos de Filosofía del Derecho 35 (2012), p. 65.

44 David Landau, Derechos sociales y límites a la reforma constitucional. La influencia de la Corte Constitucional en el derecho comparado, Bogotá, Universidad Externado de Colombia, 2015, p. 115. 


\subsection{Valoración del acceso a tratamientos experimentales bajo la perspectiva de un derecho positivo, cuando en Estados Unidos se introdujo como un derecho negativo}

En Colombia, el acceso a procedimientos experimentales fue incorporado dentro de la praxis constitucional como un débito que debe ser garantizado con los recursos del Estado, por estar excluido de los servicios asistenciales obligatorios. Esta perspectiva se enfila a que las entidades de salud, conforme a la lógica de un modelo de bienestar, tienen el deber de efectuar su prestación cuando estos insumos constituyan la última vía que puede emplearse para preservar la vida del paciente. El sistema de salud nacional tiene una fuerte tradición intervencionista, a partir de la cual, si bien se garantiza que el individuo puede elegir y decidir su propio rumbo conforme a su plan de vida, esta autonomía solo puede llevarse a cabo si se alivia la situación de carencia de los bienes indispensables para subsistir y para ejercer sus libertades. ${ }^{45}$

En Estados Unidos, el sistema sanitario tiene como fundamento el modelo liberal de mercado, por lo cual el acceso a medicamentos experimentales constituye un derecho eminentemente negativo, que se traduce en la imposibilidad de que el Estado, a través de la FDA, pueda impedir que las farmacéuticas ofrezcan estos productos a los pacientes que lo requieran. Esta diferencia entre ambos sistemas es fundamental, porque mientras en la jurisprudencia norteamericana el "derecho a intentar" incorpora una "imposibilidad de coerción", en la jurisprudencia colombiana se instituye una "obligación de prestación", lo que implica un poder de intervención sobre los actores de la salud para que garanticen el suministro de estos insumos médicos.

La Corte Constitucional introdujo entonces un deber positivo para las entidades de salud, que no estaba contemplado en la decisión originaria que se expidió en Estados Unidos, en la cual se buscaba, por el contrario, desregular el derecho de la influencia del Gobierno federal. De esa forma, se incurrió en una interpretación descontextualizada y asistemática, en la que se desconoció que la sentencia de la Corte de Apelaciones de Columbia tenía como objetivo ampliar el margen de libertad, para que los particulares y los fabricantes pudieran acceder a este tipo de medicamentos. Muy diferente al contexto local, en el cual, prácticamente, se obliga a las EPS a conceder su prestación, si se rehúsan a suministrarlos por no estar dentro del plan de beneficios.

45 Carlos Bernal Pulido, El derecho de los derechos: fundamentos, concepto y estructura de los derechos sociales, Bogotá, Universidad Externado de Colombia, 2008, p. 125. 


\subsection{Concepción del derecho bajo un criterio solidario, cuando en la perspectiva norteamericana se planteó con un enfoque individualista}

El sistema de salud colombiano tiene como fundamento una concepción solidaria, ${ }^{46}$ a partir de la cual los sectores con mayores recursos económicos deben contribuir al financiamiento de la seguridad social de las personas de bajos ingresos, y también con la subvención de aquellos que, por diversas circunstancias, están imposibilitados para procurarse su propio sustento y el de su familia ${ }^{47}$ Esta visión permite que el Estado pueda, por vía de aportes adicionales o impuestos especiales, financiar los servicios de salud, incluyendo los medicamentos o tratamientos experimentales para corregir situaciones de inequidad vital o económica. Sin embargo, en Estados Unidos, esta percepción no es aplicable en la medida que su esquema de aseguramiento tiene como fundamento un fuerte individualismo ${ }^{48}$ a partir del cual se rechaza que los contribuyentes tengan que financiar la situación deficitaria de otras personas, pues de esa forma se genera una dependencia absoluta del Gobierno, lo cual no es deseable para un modelo que garantiza la libertad de acción de los sujetos.

Cuando se incluyó el derecho a tratamientos experimentales en Estados Unidos, se hizo sobre la base de exonerar al Estado de cualquier carga fiscal para financiar estos procedimientos, de manera que únicamente pudieran ser adquiridos por aquellos individuos con capacidad económica. La filosofía de ese sistema censura que se pueda gravar a los ciudadanos para compensar el déficit social o patrimonial de otros. Esta concepción se nutre de importantes teóricos como Hayek y Nozik, que señalan que no es admisible que en forma coactiva se despoje a las personas de sus recursos, para transferirlos a otros sujetos, ya que ello conduciría a una expropiación injustificada, reflejo de un régimen colectivista o autoritario. ${ }^{49}$

46 La solidaridad parte de la convicción de que aquellos supuestos que generan un riesgo que amenaza el mínimo vital (la falta de ingresos en la vejez o en la invalidez, el súbito desempleo, la ausencia imprevista de un generador de ingresos en el hogar, una enfermedad catastrófica no anticipada) y que no pueden ser cubiertos o atenuados a través de un simple esfuerzo individual o familiar, se cubran por la vía de una contribución grupal (Leonardo Cañón Ortegón, "La solidaridad como fundamento del Estado social de derecho, de la seguridad social y la protección social en Colombia", Revista Páginas de Seguridad Social 1[2017], p. 10).

47 Corte Constitucional, Sentencia C-313 de 2014, M. P. Gabriel Eduardo Mendoza Martelo.

48 Si se analiza la visión de autores como Jhon Dewey o Walt Withman, que conceptualizaron el individualismo, se trata de una serie de libertades económicas, espirituales y jurídicas que son inherentes al ser humano, que no pueden ser interferidas por medidas del Gobierno o de terceras personas, aunque se trata de potestades limitadas por los derechos de los demás y los deberes públicos, de lo que se deriva que su aplicación es acentuada y no absoluta (Walt Whitman, Perspectivas democráticas, Buenos Aires, Editorial Americalee, 1944, pp. 30-50, y Jhon Dewey, Viejo y nuevo individualismo, Barcelona, Paidos, 2003, pp. 100-140).

49 Friedrich Hayek, Camino de servidumbre, Madrid, Alianza, 1974, pp. 75-90; Robert Nozick, Anarquía, Estado y utopía, Nueva York, Basik Books, 1974, pp. 120-150. 
Sobre este aspecto, es importante retomar la sentencia emitida por la Corte Suprema de Estados Unidos en 2012, que analizó el Obamacare, ${ }^{50}$ donde se ratificó la prohibición de que los estados federales y el Gobierno central incorporen cargas administrativas o fiscales en contra de las compañías aseguradoras, y la obliga a cubrir servicios de salud, incluidos, por supuesto, medicamentos experimentales.

A nivel nacional, la percepción es completamente distinta, en la medida que el esquema prestacional se estructura de modo que si las personas no pueden ayudarse a sí mismas o han fallado sus círculos de apoyo asociativo, el Estado debe entrar a garantizar la atención en salud, mediante acciones positivas. ${ }^{51}$

El hecho de que se haya incorporado el derecho a tratamientos experimentales bajo una óptica solidaria significa que se obliga a los asegurados del régimen contributivo a subvencionar la prestación de estos medicamentos, porque sus altos costos y su falta de acreditación científica hacen que su oferta sea muy reducida, de manera que deben ser financiados mediante esos recursos.

Por el contrario, en la idiosincrasia estadounidense se considera que la atención sanitaria es altamente onerosa y que, ante la escasez de recursos, es injusto expandir su ámbito de protección, independientemente de las circunstancias socioeconómicas de las personas, en la medida que, de esa forma, se estaría potenciando la desigualdad. ${ }^{52}$ La inclusión del derecho a tratamientos experimentales como una obligación solidaria a cargo del Estado y la sociedad resulta ser una quimera que no se compagina con la concepción individualista norteamericana, que se contrapone al altruismo fiscal y a la colectivización de los gastos.

\subsection{Inclusión del derecho en el marco de un enfoque de intermediación que se opone al modelo de independencia médica norteamericano}

La independencia médica se basa en que el profesional de la salud esté en plena libertad y capacidad de emitir su opinión acerca de cuál es el tratamiento más

50 Corte Suprema de Justicia de Estados Unidos, Caso Obamacare, Sentencia de 28 de junio de 2012. Es importante anotar que el Obamacare, "Ley de Protección del Paciente y de Cuidado Asequible de la Salud", tuvo como objetivo establecer un sistema de cobertura universal de salud, a partir de cuatro mecanismos: a) la reducción de las primas que cobran las aseguradoras; b) la obligación de las personas de adquirir una póliza, bajo la coacción de multas; c) el control de cláusulas abusivas que pudieran discriminar a los usuarios; d) la ampliación del Medicare a personas de bajos ingresos, diferentes de los mayores de 65 años, los nativos, los veteranos y los discapacitados (Katherine Record, "Litigating the ACAACA: securing the right to health within a framework of negative right", Review American Journal of Law and Medicine 38 [2012], p. 542).

51 Rodolfo Arango, "Justiciabilidad de los derechos sociales fundamentales en Colombia: aporte a la construcción de un ius constitutionale commune en Latinoamérica", Revista de Derecho Público 12 (2001), p. 25. Según la Corte Constitucional, "los individuos son los primeros convocados a proveerse aquellos servicios médicos que se encuentran excluidos y solo en aquellos casos en que carezcan de recursos económicos suficientes para tal fin, procede la intervención del Estado para garantizar la efectiva concreción del derecho a la salud, proporcionando los servicios no cubiertos con cargo a recursos públicos" (Corte Constitucional, Sentencia T-438 de 2009, M. P. Gabriel Eduardo Mendoza Martelo).

52 Allen Buchanan, "A right to a decent minimum of health care", Philosophy and Public Affairs 13 (1984), p. 63. 
adecuado para sus pacientes. ${ }^{53} \mathrm{Si}$ se analiza el concepto anterior, es posible avizorar que en Colombia se presenta un déficit importante en esta materia, pues los profesionales de la salud se encuentran sujetos a controles externos que pueden desconocer o limitar sus órdenes médicas. ${ }^{54}$ Estos órganos obstaculizan el acceso al derecho a tratamientos experimentales, pues se encuentran facultados para oponerse a la opinión de los galenos que recomienden el uso de estos procedimientos, por lo que pueden impedir o paralizar su suministro a los pacientes.

El artículo 12 de la Resolución 1328 de 2016 estableció que para la aprobación de procedimientos de experimentación no basta con la orden del médico tratante, sino que se requiere que una junta médica emita un concepto favorable y que su decisión negativa o positiva se registre en un aplicativo en línea del Ministerio de Salud.

Si el paciente se encuentra en una situación de riesgo vital, como suele ocurrir cuando se acude a tratamientos experimentales, es posible omitir el requerimiento a la junta médica, pero después de los cinco días siguientes al suministro del medicamento o tecnología, se debe acudir a este organismo para que confirme o desautorice la continuidad del servicio al paciente. La orden que imparte el profesional de la salud es totalmente falible y puede ser anulada en cualquier momento, incluso desconociendo la Sentencia T-057 de 2015, en la que se prohíbe, para casos futuros, la suspensión de estos tratamientos, al ser considerados como la última vía de auxilio vital.

En el derecho norteamericano no existe ningún órgano intermediario que pueda limitar la facultad de ordenación médica, pues no hay instancias científicas o administrativas que puedan revocar o impedir las prescripciones de los galenos. La FDA solo tiene un rol de intervención mínima que le permite impedir la circulación de medicamentos cuando no superen la primera fase de experimentación o se haya demostrado que generan daños, pero esa potestad limitativa solo se puede ejercer frente a las compañías farmacéuticas y no frente al personal de la salud.

En el ámbito colombiano, la situación es totalmente diferente, pues el modelo sanitario limita las apreciaciones científicas de los médicos a través de instancias intermedias, con la finalidad de evitar que, mediante sus conceptos, se ordenen gastos muy onerosos o se prescriban medicamentos que no tengan un margen de eficacia o seguridad debidamente acreditado. Esta perspectiva reduccionista de las facultades médicas busca, principalmente, proteger los de 2015, Medellín, Universidad de San Buenaventura, 2016, p. 57. 
fondos de las entidades aseguradoras, aunque ello derive en una atención deficitaria en salud que contravenga los intereses de los pacientes. ${ }^{55}$

En Colombia, las restricciones al ejercicio de la lex artis médica son un rezago de un modelo paternalista que todavía no se ha podido superar, con efectos nocivos sobre la libertad del médico y la autonomía del paciente, a quien por efecto de unas entidades externas, se le afecta su decisión de someterse al tratamiento experimental.

El sistema norteamericano se opone a cualquier tipo de intermediación que afecte la autonomía de los galenos, y, en ese sentido, la inclusión del acceso a tratamientos experimentales en Colombia se realizó en el marco de un esquema prestacional que limita ampliamente la potestad de los profesionales para su ordenación libre y sin injerencias.

\subsection{Introducción del derecho en el contexto de un mercado regulado, cuando en Estados Unidos se incluyó en un mercado desregulado}

Un aspecto fundamental del sistema de salud norteamericano es su tendencia a la desregulación de todos los actores (compañías farmacéuticas, entidades de salud y consumidores) que lo integran. ${ }^{56} \mathrm{El}$ Gobierno federal no puede intervenir en la esfera de la libertad de las personas, compeliéndolas a obtener un aseguramiento médico, ni tampoco puede obligar a las aseguradoras a ofrecer coberturas de medicamentos experimentales cuando consideren que no son convenientes para sus finanzas.

El comercio de servicios sanitarios norteamericano tiene como base esencial un criterio de autorregulación, ${ }^{57}$ a partir del cual son los actores involucrados los que establecen las reglas a las que van a sujetarse en el tráfico mercantil, en procurando del equilibrio entre la oferta y la demanda. Estados Unidos le apuesta más a medidas como alivios o créditos fiscales para incentivar la oferta y la demanda de servicios médicos experimentales que a acciones represivas o sancionatorias.

55 José Patiño Restrepo, “La atención de la salud en un modelo de aseguramiento comercial”, Revista Colombiana de Cirugía 26 (2011), p. 10.

56 Irene Guijarro Sobrino, "La reforma sanitaria de Obama: limitado avance en la protección del derecho a la salud", Revista Española de Derecho Constitucional 101 (2014), p. 207.

57 La autorregulación implica que la actuación del Estado debe ser promover la libre competencia y no limitarla mediante monopolios en la oferta o la demanda de bienes y servicios. Se trata de una visión de la economía que pugna con el establecimiento de controles fiscales, en la medida que pueden producir inflación y desincentivar la iniciativa privada (Milton Friedman, La economía monetarista, Barcelona, Gedisa, 2010, p. 132). 
En Colombia, por el contrario, hay una tendencia creciente a aplicar un criterio de fiscalización ${ }^{58}$ a los actores que se encuentran inmersos dentro del sistema, cuando no cumplen con las cargas administrativas que se les imponen, a partir de figuras punitivas o coercitivas. A nivel nacional existe un importante componente sancionatorio que faculta a la Superintendencia de Salud para imponer fuertes medidas coactivas cuando las EPS deniegan el suministro de un medicamento, incluso si se trata de naturaleza experimental, o cuando dilatan su entrega por más de 48 horas, contadas desde que la persona lo reclama de manera formal. ${ }^{59}$

Esta situación de contraste entre ambos sistemas de salud, demuestra claramente que la Corte Constitucional no tuvo en cuenta el contexto en el cual se incorporó el derecho a acceder a tratamientos experimentales dentro del ordenamiento norteamericano, pues en ese país lo que se buscó fue desregular el intercambio de estos insumos no convencionales, de manera que los clientes pudieran negociar su accesibilidad directamente con las farmacéuticas, mientras que, en Colombia, su introducción fue el resultado de una profundización de la fiscalización del Estado en el mercado sanitario, pues se obligó a las EPS a ofrecer un producto que no se encuentra dentro de su plataforma de servicios y que, en circunstancias normales de competencia, no hubieran financiado con sus recursos.

En ese sentido, mientras en Estados Unidos se pretendió que las comercializadoras de medicamentos y los usuarios pudieran definir las condiciones de competencia para la provisión del producto, en Colombia, lo que hizo la Corte fue irrumpir como un tercero dentro de las relaciones de mercado, para definir bajo qué condiciones se debían suministrar los medicamentos experimentales, sin tener en cuenta si los actores de la salud estaban dispuestos a garantizar su cobertura.

\section{Conclusiones}

1. En Estados Unidos, el derecho a acceder a tratamientos experimentales se introdujo como un derecho negativo, bajo la perspectiva de que el Estado, a través de la FDA, no podía limitar su suministro por parte de las farmacéuticas, mientras en Colombia se incorporó como un derecho positivo, es decir como un débito a cargo de los recursos públicos del Estado. La Corte Consti-

$58 \quad$ La fiscalización hace alusión a una injerencia profusa sobre determinados mercados que el Gobierno califica de interés público, para evitar la conformación de monopolios, contrarrestar prácticas de competencia desleal y controlar los precios de los bienes que se transan (Eugenio Rivera, "Teorías de la regulación en la perspectiva de las políticas públicas", Gestión y Política Pública 2 [2004], p. 334).

59 Los valores que pueden alcanzar las sanciones pecuniarias por falta de suministro oportuno de medicamentos se encuentran establecidos en el artículo 19 de la Resolución 3140 de 2011 del Ministerio de Salud, donde se señala que las multas a los representantes legales de las entidades prestadoras pueden alcanzar entre los 100 y los $200 \mathrm{SMLV}$, mientras que si se trata de la persona jurídica que suministra los servicios clínicos u hospitalarios, la penalidad puede ascender a la suma exorbitante de $2.500 \mathrm{SMLV}$. 
tucional interpretó el derecho como una "obligación de prestación" a cargo de las entidades de salud, mientras en Norteamérica se valoró como una "imposibilidad de coerción" a los particulares.

2. En el sistema sanitario estadounidense la financiación de los tratamientos experimentales la debe realizar el paciente con sus propios recursos, en la medida que ese esquema prestacional se fundamenta en un fuerte individualismo que presume que las personas cuentan con la solvencia económica para adquirir estos productos médicos, mientras en Colombia la subvención de estos procedimientos con base en la solidaridad se endilgó al Estado a través de la Administradora de los Recursos del Sistema General de Seguridad Social en Salud (ADRES).

3. En el ordenamiento norteamericano el acceso a medicamentos experimentales no se encuentra restringido por órganos administrativos o técnicos que limiten la independencia científica del médico tratante para su ordenación a los pacientes, lo que contrasta con el sistema de salud nacional que, a través de la Resolución 1328 de 2016, faculta a una serie de juntas médicas que figuran como mecanismos de intermediación para aprobar o denegar la prestación de estos servicios, lo que atenta contra la autonomía de los profesionales de la salud que prescriben estos insumos médicos.

4. La inserción del derecho a tratamientos experimentales se realizó forma descontextualizada y asistemática, con desconocimiento de los fundamentos que estructuran el sistema sanitario estadounidense, en el cual hay una autorregulación de los actores de la salud para su prestación, no existe obligación de aseguramiento universal ni de subvención fiscal y se garantiza un amplio margen de libertad a los particulares en la oferta y demanda de servicios de salud; por el contrario, en el esquema prestacional colombiano hay una fuerte fiscalización de los fabricantes y los aseguradores de salud en el suministro de estos insumos médicos, donde se puede obligar a las EPS a financiar estos procedimientos y los recursos tienen que ser reembolsados por el ADRES.

5. En Colombia existe un vacío legislativo para regular el acceso a tratamientos experimentales, pues la Resolución 8430 de 1993, expedida por el Ministerio de Salud, es un instrumento normativo anacrónico y defectuoso que se encuentra muy distante de una verdadera legislación sólida que regule aspectos esenciales como el consentimiento cualificado, los tipos de enfermedades que puedan ser objeto de estos procedimiento, los casos en los cuales debe excluirse su suministro, los destinatarios de estos insumos médicos y la responsabilidad que le puede caber a los profesionales de salud por el empleo de estos elementos.

\section{Bibliografía}

Amaya, Amalia, "La coherencia en el derecho", en DOXA, Cuadernos de Filosofía del Derecho 35 (2012), pp. 59-90. DOI: https://doi.org/10.14198/doxa2012.35.04 
Arango, Rodolfo, "Justiciabilidad de los derechos sociales fundamentales en Colombia: aporte a la construcción de un ius constitutionale commune en Latinoamérica", Revista de Derecho Público 12 (2001) pp. 17-34, en https:// archivos.juridicas.unam.mx/www/bjv/libros/7/3063/4.pdf

Arrieta, Alejandro, "Seguro de salud y principio contributivo de la seguridad social en los Estados Unidos de América", Revista Latinoamericana de Derecho Social 23 (2016), pp. 3-30. DOI: https://doi.org/10.1016/j.rlds.2016.08.001

Banting, Keith y Stand Corbett, Health Policy and Federalism. A comparative perspective on Multi-Level Governance, New York, Queen's University, 2002.

Bellamy, Jann, "The illusions of 'right to try' laws", Review Science-Based Medicine, 2014, en https://sciencebasedmedicine.org/the-illusions-of-right-totry-laws/, fecha de consulta: 17 de enero de 2020.

Bernal Pulido, Carlos, El derecho de los derechos: fundamentos, concepto y estructura de los derechos sociales, Bogotá, Universidad Externado de Colombia, 2008.

Buchanan, Allen, "A right to a decent minimum of health care", Philosophy and Public Affairs 13 (1984), pp. 55-78, en https://www.jstor.org/stable/2265199

Bustamante Rojas, Carlos, Fases del desarrollo de un nuevo medicamento. Ponencia del Programa de Especialización en Farmacología Clínica, Bogotá, Universidad de La Sabana, 2013.

Cañón Ortegón, Leonardo, "La solidaridad como fundamento del Estado social de derecho, de la seguridad social y la protección social en Colombia", Revista Páginas de Seguridad Social 1 (2017), pp. 5-29. DOI: https://doi. org/10.18601/25390406.n1.01

Ciaglo, Michael, "Quicker access to experimental drugs", The New York Times, 2015, en https://www.nytimes.com/2015/02/12/opinion/quicker-access-toexperimental-drugs.html, fecha de consulta: 17 de enero de 2020.

Congreso de la República, Ley 1751 de 2015, "Por medio de la cual se regula el derecho fundamental a la salud y se dictan otras disposiciones", Diario Oficial 49.427 de 16 de febrero de 2015.

Corte Constitucional, Auto 035 de 2009, M. P. Manuel José Cepeda Espinosa.

Corte Constitucional, Sentencia C-313 de 2014, M. P. Gabriel Eduardo Mendoza Martelo.

Corte Constitucional, Sentencia T-057 de 2015, M. P. Martha Victoria Sáchica Méndez.

Corte Constitucional, Sentencia T-180 de 2013, M. P. Jorge Ignacio Pretelt Chaljub. 
Corte Constitucional, Sentencia T-303 de 2016, M. P, Jorge Ignacio Pretelt Chaljub.

Corte Constitucional, Sentencia T-438 de 2009, M. P. Gabriel Eduardo Mendoza Martelo.

Corte Constitucional, Sentencia T-760 de 2008, M. P. Manuel José Cepeda Espinosa.

Corte Constitucional, Sentencia T-901 de 2009, M. P. Humberto Antonio Sierra Porto.

Corte de Apelaciones del Distrito de Columbia, caso Abigail Alliance for Better Access to Developmental Drugs and Washington Legal Foundation, appellants vs. Eschenbach, sentencia del 7 agosto de 2007.

Corte Suprema de Justicia de Estados Unidos, caso Obamacare, sentencia del 28 de junio de 2012.

Dewey, Jhon, Viejo y nuevo individualismo, Barcelona, Paidos, 2003.

Duque Giraldo, Mateo, "Precedente constitucional en materia de 'derecho a ser intentado' o 'right to try' para tratamientos experimentales", Revista CES de Derecho 1 (2018), pp. 143-163. http://dx.doi.org/10.21615/cesder.9.1.8

Freidson, Eliot, La profesión de la medicina, Chicago, Universidad de Chicago, 1988.

Friedman, Milton, La economía monetarista, Barcelona, Gedisa, 2010.

Gañan Echavarría, Jaime León, “De la naturaleza jurídica del derecho a la salud en Colombia", Revista RIDE de la Biblioteca digital de la Superintendencia de Salud 3 (2013), pp. 7-19, en https://www.minsalud.gov.co/sites/rid/ Lists/BibliotecaDigital/RIDE/IA/SSA/naturaleza-juridica-derecho-saludcolombia.pdf

Giraldo, Óscar y José Corral, Autonomía médica de la ley 100 de 1993 al nuevo panorama de la ley estatutaria 1751 de 2015, Medellín, Universidad de San Buenaventura, 2016.

Guijarro Sobrino, Irene, "La reforma sanitaria de Obama: limitado avance en la protección del derecho a la salud", Revista Española de Derecho Constitucional 101 (2014), pp. 181-212, en https://www.jstor.org/stable/24887272?seq=1

Hayek, Friedrich, Camino de servidumbre, Madrid, Alianza, 1974.

Herrera, Carlos Miguel, "El concepto de solidaridad y sus problemas políticoconstitucionales. Una perspectiva iusfilosófica", Revista de Estudios Sociales 46 (2013), pp. 63-73, en https://journals.openedition.org/revestudsoc/7802

Hirsch, Ran, Comparative Matters, The Renaissance of Comparative Constitutional Law, Oxford University Press, 2014. 
Hogan, Emily, "Legislación del derecho intentar y sus implicaciones para el proceso de aprobación de drogas de la FDA", Revista de Derecho y Política 50 (2016), pp. 171-190, en https://openscholarship.wustl.edu/law_journal_ law_policy/vol50/iss1/8/

Iriart, Celia, "El sistema de salud de los Estados Unidos: mitos y realidades", Revista Saúde em Redes 1 (2016), pp. 125-139. doi: http://dx.doi. org/10.18310/2446-4813.2016v2n2p125-139

Landau, David, Derechos sociales y límites a la reforma constitucional. La influencia de la Corte Constitucional en el derecho comparado, Bogotá, Universidad Externado de Colombia, 2015.

Lopera, María Mónica, “Revisión comentada de la legislación colombiana en ética de la investigación en salud", Revista Biomédica 37 (2017), pp. 577-589 en https://doi.org/10.7705/biomedica.v37i4.3333

Lowes, Robert, "Right-to-try gains steam as FDA eases compassionate use", Medscape Medical News, 2017, en https://www.medscape.com/viewarticle/886525, fecha de consulta: 17 de enero de 2020.

Macho, Ana Belén, "La reforma de la financiación del sistema sanitario en Estados Unidos. ¿Es la affordable care act ("obamacare") relevante para Europa?", Revista Tax and Public Finance Law Seminar Series 1 (2015), pp. 2-32, en https://repositori.upf.edu/bitstream/handle/10230/25883/TaxPublicFinanceLaw-1.pdf? sequence $=1$ \&isAllowed $=y$

Ministerio de Salud, Resolución 8430 de 1993, "Por la cual se establecen las normas científicas, técnicas y administrativas para la investigación en salud", en https://www.minsalud.gov.co/sites/rid/Lists/BibliotecaDigital/ RIDE/DE/DIJ/RESOLUCION-8430-DE-1993.PDF

Ministerio de Salud, Acuerdo 008 de 1994, "Por el cual se adopta el Plan Obligatorio de Salud para el Régimen Contributivo del Sistema General de Seguridad Social en Salud", en https://www.minsalud.gov.co/sites/rid/ Lists/BibliotecaDigital/RIDE/DE/DIJ/ACUERDO-08-DE-1994.pdf, fecha de consulta: 17 de enero de 2020 .

Ministerio de la Salud, Resolución 1328 de 2016, “Por la cual se establece el procedimiento de acceso, reporte de prescripción, garantía del suministro, verificación, control, pago y análisis de la información de servicios y tecnologías en salud no cubiertas por el Plan de Beneficios en Salud con cargo a la UPC y se dictan otras disposiciones", en https://www.minsalud.gov. co/Normatividad_Nuevo/Resoluci\%C3\%B3n\%201328\%20de\%202016.pdf 
Naciones Unidas, Pacto de Derechos Civiles y Políticos. Ratificado por la Asamblea General en su Resolución 2200 A (XXI) de 16 de diciembre de 1966.

Nozick, Robert, Anarquía, Estado y utopía, Nueva York, Basik Books, 1974.

Organización Panamericana de la Salud, Perfil de sistemas y servicios de salud en Estados Unidos de América, Columbia, Editorial Panamericana, 2018.

Patiño Restrepo, José, "La atención de la salud en un modelo de aseguramiento comercial", Revista Colombiana de Cirugía 26 (2011), pp. 9-10, en http:// www.scielo.org.co/pdf/rcci/v26n1/v26n1a1.pdf

Ramírez, Andrés, David Beltrán Rocha, Luis Suárez Durango, Sara Álvarez Rodríguez, "Implicaciones de la Ley Estatutaria 1751 de 2015 sobre las entidades promotoras de salud del régimen contributivo", Revista CES de Derecho 2 (2016), pp. 13-41, en http://www.scielo.org.co/pdf/cesd/v7n2/ v7n2a03.pdf

Record, Katherine, "Litigating the ACAACA: securing the right to health within a framework of negative right", Review American Journal of Law and Medicine 38 (2012), pp. 537-547. DOI: https://doi.org/10.1177/009885881203800211

Rivera, Eugenio, "Teorías de la regulación en la perspectiva de las políticas públicas", Gestión y Política Pública, 2 (2004), pp. 309-372, en https://www. redalyc.org/articulo.oa?id=13313201

Trajano, Augustus y Sonia Fleury, "La reforma del sistema de salud de los Estados Unidos de América en los años 90", Revista Salud Colectiva 1 (2005), pp. 129-153, en https://www.scielosp.org/article/scol/2005.v1n2/129-153/

Turkewitz, Julie, "Patients seek 'right to try' new drugs", The New York Times, 2015, en https://www.nytimes.com/2015/01/11/us/patients-seek-right-totry-new-drugs.html, fecha de consulta: 17 de enero de 2020.

Whitman, Walt, Perspectivas democráticas, Buenos Aires, Editorial Americalee, 1944. 